\title{
Transgenerational effects enhance specific immune response in a wild passerine
}

Juli Broggi, Ramon C. Soriguer, Jordi Figuerola

Vertebrate mothers transfer diverse compounds to developing embryos that can affect their development and final phenotype (i.e. maternal effects). However, the way such effects modulate offspring phenotype, in particular their immunity remains unclear. To test the impact of maternal effects on offspring development we treated with Newcastle disease virus (NDV) vaccine wild breeding house sparrows (Passer domesticus) in Sevilla, SE Spain. Female parents were vaccinated when caring first broods eliciting a specific immune response to NDV. The immune response to the same vaccine, and to the PHA inflammatory test were measured in 11-day-old chicks from their following brood. Vaccinated chicks from vaccinated mothers developed a stronger specific response that was related to maternal NDV antibody concentration while rearing their chicks. Chick's carotenoid concentration and total antioxidant capacity in blood were negatively related to NDV antibody concentration, whereas no relation with PHA response was found. Specific NDV antibodies could not be detected in 11-day-old control chicks from vaccinated mothers, implying that maternally transmitted antibodies are not directly involved but may promote offspring specific immunity through a priming effect, while other immunity components remain unaffected. Maternally transmitted antibodies in the house sparrow are short-lived, depend on maternal circulation levels and enhance pre-fledging chick specific immunity when exposed to same pathogens as the mothers. 
3 Juli Broggi ${ }^{1,2,4}$, Ramón C. Soriguer ${ }^{1,3}$ and Jordi Figuerola ${ }^{1,3}$

4 1. Estación Biológica Doñana, CSIC. Avda. Americo Vespucio s/n. 41092 Sevilla, Spain.

5 2. Research Unit of Biodiversity (UO, CSIC, PA), Universidad de Oviedo, 33600 Mieres, Spain.

6 3. CIBER Epidemiología y Salud Pública (CIBERESP), Spain

7 4. Author for correspondence. Department of Biology, Section of Evolutionary Ecology,

8 University of Lund, S-22362 Lund, Sweden. Email: julibroggi@gmail.com

9 Running title: Maternal effects on passerine immunity

\section{TRANSGENERATIONAL EFFECTS ENHANCE SPECIFIC IMMUNE RESPONSE IN}

\section{A WILD PASSERINE}

Key-words: Antibodies; Immune priming; Newcastle disease Virus; Oxidative stress; Passer domesticus

Author Contributions: JB, JF and RCS conceived and designed the experiments. JB performed the experiments. JB and JF analyzed the data. JB wrote the manuscript; other authors provided editorial advice.

Compliance with Ethical Standards: The authors declare no conflicts of interest, and that none of the material presented in this manuscript has been published or is under consideration elsewhere. This submission for publication has been approved by all relevant authors and institutions; and all persons entitled to authorship have been so named. All authors have seen and agreed to the submitted version of the manuscript. All procedures followed the guidelines of the Estación Biológica de Doñana, CSIC ethical committee and the regional government for the manipulation of birds and collection of blood samples (N/RefS.:G YB/AFR/CMM). All research presented in the manuscript was conducted in accordance with all applicable laws and rules set forth by their governments and institutions. 


\section{ABSTRACT}

27 Vertebrate mothers transfer diverse compounds to developing embryos that can affect their

28 development and final phenotype (i.e. maternal effects). However, the way such effects modulate offspring phenotype, in particular their immunity remains unclear. To test the impact of maternal effects on offspring development we treated with Newcastle disease virus (NDV) vaccine wild breeding house sparrow (Passer domesticus) in Sevilla, SE Spain. Female parents were vaccinated when caring first broods eliciting a specific immune response to NDV. The immune response to the same vaccine, and to the PHA inflammatory test were measured in 11-day-old chicks from their following brood. Vaccinated chicks from vaccinated mothers developed a stronger specific response that was related to maternal NDV antibody concentration while rearing their chicks. Chick's carotenoid concentration and total antioxidant capacity in blood

37 were negatively related to NDV antibody concentration, whereas no relation with PHA response was found. Specific NDV antibodies could not be detected in 11-day-old control chicks from vaccinated mothers, implying that maternally transmitted antibodies are not directly involved but

40 may promote offspring specific immunity through a priming effect, while other immunity components remain unaffected. House sparrow maternally transmitted antibodies are short-lived,

42 depend on maternal circulation levels and enhance pre-fledging chick specific immunity when exposed to same pathogens as the mothers. 
46

47

\section{INTRODUCTION}

Vertebrate mothers may provide a favourable growing environment and care, but additionally can transmit diverse components such as hormones (Groothuis and Schwabl 2008), antioxidants (e.g. Royle et al 2003) or immunoglobulins (Grindstaff et al 2003; Boulinier and Staszewski 2008; Hasselquist and Nilsson 2009) that can have important phenotypic consequences on the developing embryo (Mousseau et al 2009). These maternal effects constitute a major source of transgenerational phenotypic plasticity that can vary according to environmental heterogeneity (e.g. Morosinotto et al 2013) and maternal condition (Boulinier and Staszewski 2008). However, the way these transferred components interact with each other, and modulate the development of the offspring phenotype remains unclear (Mousseau et al 2009).

The vertebrate humoral immune response targets specific infectious agents by means of antibodies $(\mathrm{Ab})$, and persists as immune memory allowing a faster response upon re-exposure to the same antigen. While adult birds can develop a humoral response in a few days (Iseri \& Klasing 2013), newborn individuals require some time to fully develop such capabilities and may rely on maternally transferred $\mathrm{Ab}$ while they develop their own endogenous response (Hasselquist et al 2012). Altricial avian small species are particularly vulnerable at hatching as they need to develop fast enough to be able to fledge in a few days time, and thus could be expected to rely preferentially on maternally transmitted Ab to develop their specific immunity. However, studies on the influence of maternal $\mathrm{Ab}$ on host development and fitness are scarce (Boulinier and Staszewski 2008), despite the important influence individual immune condition exerts on pathogen susceptibility in wild organism populations (e.g. Hochachka and Dhondt 2000). The influence of maternal $\mathrm{Ab}$ on the fitness and disease epidemiology of neonates in 
68 humans and domestic mammals is well documented, providing the offspring with transient

69 immunity against the microbial infections that the mother has encountered (Hasselquist and

70 Nilsson 2009). In avian species yolk-transmitted maternal Ab detectability ranges from days to

71 few months after hatching, depending on the species (Garnier et al 2012) and the initial amount

72 transferred (Grindstaff 2010). However, whether maternally transmitted Ab are enhancing the

73 chick's subsequent response by a priming mechanism (e.g. Grindstaff et al 2006; Reid et al

74 2006), or blocking the chick's endogenous response (e.g. Staszewski et al., 2007; Elazab et al

75 2009; Staszewski and Siitari 2010) remains poorly understood.

76 Individuals exhibit substantial differences in their immune responses and rarely develop it

77 maximally, which suggests there are some costs associated that individuals manage differently

78 (Viney et al 2005). Such individual variation in immune investment may arise from a variety of

79 factors, not only adaptive adjustments but also constraints resulting from allocation conflicts

80 with other physiological and life-history traits (Ardia et al 2011). Furthermore, individuals may

81 invest differentially in various components of the immune system (Lazzaro and Little 2009),

82 depending on the pathogen identity (Adamo 2004) or constraining nutritional or energetic factors

83 (Hõrak et al 2006), or alternatively these could vary in concert (Ardia 2005; Ahmed et al 2007),

84 but see (Forsman et al 2008).

85 Oxidative balance and carotenoids have been acknowledged among the factors mediating

86 variation in the immune responses (Hasselquist and Nilsson, 2012). The intensity of the immune

87 response is generally coupled with a shift in the oxidative balance and a decrease in carotenoid

88 concentration that are considered detrimental for the individual (Ardia et al 2011). Oxidative

89 stress is generated as a metabolic by-product resulting in damage to cell macromolecules

90 (Dowling and Simmons 2009). Organisms balance their oxidative stress by acquiring and 
91 producing antioxidants, and although most antioxidative activity is enzymatic, non-enzymatic

92 antioxidants also play a relevant role in maintaining oxidative balance, particularly in blood

93 (Cohen and McGraw 2009). Immunity costs in terms of oxidative balance have been argued to

94 underlie trade-offs between immunity and life-history traits, either by directly increasing

95 oxidative stress (Costantini and Møller 2009) or by competing for antioxidants (Monaghan et al

96 2009), but see (Speakman and Garratt 2014).

97 Carotenoids are a diverse group of lipophilic molecules important for immunity and individual

98 fitness, and since they cannot be synthesized de-novo by animals, necessarily need to be ingested

99 or acquired during embryogenesis through maternal transfer (Pérez-Rodríguez 2009). Avian

100 mothers transmit carotenoids and antioxidants through the egg yolk, and after hatching through

101 diet (Blount et al 2003; McGraw and Ardia 2003). These components interact synergistically

102 (Bédécarrats and Leeson 2006; Koutsos et al 2007) and likely stimulate the development of the

103 offspring own immune phenotype (Simons et al 2012). However, little is known on how such

104 maternal effects may interact with each other, especially on wild and non-model species

105 (Hasselquist et al 2012).

106 In this study we explored whether maternal effects modulate offspring specific immune response

107 in a wild breeding house sparrow (Passer domesticus, Linnaeus 1758) population. Using a

108 vaccine to elicit an immune response to a viral antigen we analysed how maternal exposure

109 during first brood affects transmission of antibodies to the following brood, their offspring

110 development and immune response when exposed to the same antigen during the critical post-

111 hatching period before fledging. We additionally measured chick PHA-induced inflammatory

112 response, to find out whether mother immune condition affected their offspring specific humoral

113 response, or affected more aspects of offspring immunity. Furthermore, we studied the effect of 
114 other maternally-transmitted components (antioxidative and carotenoids) which are likely to

115 affect the development of the offspring immune phenotype. We predict vaccinated mothers to 116 positively affect their offspring specific immunity, and this effect to be enhanced by higher

117 levels of maternal antioxidants, antioxidative capacity and carotenoids in blood. 


\section{MATERIALS AND METHODS}

Study area and model species

The house sparrow is a small-sized (20gr) granivorous passerine that breeds in close association with humans. House sparrows are commonly exposed to a variety of pathogens in the wild, including several epizootic pathogens resulting from the close association with humans and livestock e.g. Newcastle Disease Virus or Salmonella (Anderson 2006). The Newcastle Disease Virus (NDV) is a worldwide distributed avian paramyxovirus that causes a highly contagious disease, representing a severe problem for the poultry industry and also wild fauna (Alexander 2009). The virus is circulating in the study area as NDV antibodies were detected previously in 11 out of 81 individuals analyzed (Broggi et al 2013).

The study area is located in a private land surrounded by farmland and mixed forest, la Cañada de los Pájaros $\left(37^{\circ} 14^{\prime} \mathrm{N}, 6^{\circ} 07^{\prime} \mathrm{W}\right)$ in Sevilla, SE Spain. The study population is about 100 pairs breeding naturally in wooden nestboxes at an average height of $2 \mathrm{~m}$ and within an area of 10 ha. Females lay up to 4 broods per year, usually in the same nestbox, with an average clutch of 4.5 eggs that hatch asynchronously. Chicks fledge at the age of 12-15 days if undisturbed. Breeding season starts in early April and lasts until the end of August (own unpublished data).

\section{Experimental approach}

From April $1^{\text {st }}$ (the beginning of the breeding season) until August 2010, nestboxes were checked every second day to record breeding parameters. Breeding females were captured at the nest when chicks were older than 8 days to prevent nest desertion. Newly captured females were randomly assigned to the treatment (subcutaneous injection of $0.2 \mathrm{ml}$ of a commercial 
142 inactivated NDV vaccine HIPRAVIAR ${ }^{\circledR}$ BPL2) or a control group (injection with $0.2 \mathrm{ml}$ of

143 PBS), following the results of a pilot study in the same population (Broggi et al 2013). Before

144 treatment, blood was sampled from the jugular vein $(0.2 \mathrm{ml})$ and kept cool $\left(\sim 4^{\circ} \mathrm{C}\right)$ for less than

$14512 \mathrm{~h}$ before centrifugation (20 min at $4000 \mathrm{rpm}$ ). Cellular phase and sera were stored separately at

$146-20^{\circ} \mathrm{C}$ for later analyses (see below), and wing, tarsi and body mass were measured. Females

147 were allowed to complete the first breeding attempt without further manipulation to minimise

148 disturbance. Recaptured females were treated as in their first capture. During the next breeding

149 attempt the chicks were weighed (to $0.1 \mathrm{~g}$ ) on their $4^{\text {th }}$ day of age, and were inoculated

150 subcutaneously with either NDV vaccine $(0.1 \mathrm{ml})$ or a control treatment (PBS). Chicks within

151 each brood were ordered by body mass and inoculated alternatively with vaccine or control

152 treatment, switching the starting treatment in each different brood. Chicks were recognized by

153 innocuous paint in their claw, and were repainted until they were marked with aluminium rings

154 when $\sim 6$ days of age. Due to hatching asynchrony, chick age differed within broods in up to 4

155 days (JB pers.obs.). When average brood age was 11 days, chicks were weighed, and their tarsi

156 and wing length measured (to $0.1 \mathrm{~mm}$ ). Blood samples were taken from the chick's jugular vein

$157(0.1 \mathrm{ml})$ and processed as with adult female samples. Finally, chicks were subjected to a

158 phytohaemagglutinin (hereafter PHA) immune challenge before being released in their nestbox.

159 On the following day, chicks were re-measured (see below for details on the PHA immune

160 challenge). Sex of the chicks was determined by molecular techniques based on DNA obtained

161 from blood samples (Fridolfsson and Ellegren 1999). Females were recaptured on the second

162 breeding attempt, and blood was sampled to measure blood metabolites and NDV-Ab

163 concentration (see below). Each experimental female was included once in the dataset, and

164 whenever captured in more than two consecutive nesting events ( 2 cases), only the last breeding 
165 episode was included in order to ensure a high female response to vaccination at the time of egg-

166 laying. On average, experimental females were challenged 3 to 10 weeks before egg-laying of

167 the following clutch, well-within the antibody circulation peak after vaccination (Broggi et al 168 2013; Midamegbe et al 2013). Altogether the dataset consisted of 88 chicks from 25 different 169 females (13control vs. 12 vaccinated). Control chick sample size consisted of 21 individuals 170 originating from control mothers and 19 from vaccinated mothers. Vaccinated chick sample size 171 consisted of 25 individuals originating from control mothers and 23 originating from vaccinated 172 mothers (Table 1).

Immunological measurements

175 We used haemagglutination inhibition test (HI) to assess NDV-Ab concentration in sera. The test sera $(25 \mu \mathrm{l})$ were sequentially diluted in PBS from 1/2 to $1 / 640$ and $4 \mathrm{HA}$ units of antigen HIPRAVIAR ${ }^{\circledR}$-CLON E.Newcastle, clon CL/79 were added to each dilution. The mixture was added to $50 \mu 1$ of chicken RBC's and after 30 minutes at room temperature checked for agglutination. NDV-Ab concentration was scored as the highest dilution where agglutination was observed. We used commercial positive and negative controls (VLDIA053 HAR-NDL, NDV strain La Sota and VLDIA030 SPF-CH-Chicken negative), further details can be found in Broggi et al (2013). None of the females at first capture nor control females at second capture presented NDV titers higher than $1 / 8$, the lower limit to consider a serum positive to NDV according to standard laboratory practices (OIE 2015).

At 11 days of age, chicks were challenged with PHA in the patagia. PHA is a mitogen of vegetal origin that when injected intradermally induces an immune response mainly reflecting individual pro-inflammatory potential (Vinkler et al 2010). Birds were injected in the right wing web with 
$18850 \mu 1$ of 5:2 PHA-P (L-8754 Sigma-Aldrich) in PBS following Smits et al (1999). Patagium

189 width was measured at the point of injection (to the nearest $0.01 \mathrm{~mm}$ ) just prior to and at least 24

$190 \mathrm{~h}$ from challenge, using a pressure sensitive micrometer (Baxlo Precision S.L.), and the

191 difference was used as PHA response thereafter. Time between measurements averaged

$19240.20 \pm 0.81$ hours, range $=25-45$ after challenge. PHA-challenge elicits an inflammatory response

193 that reaches its maximum after $\sim 6 \mathrm{~h}$ from injection, and can last up to 72 hours (Navarro et al.

194 2003). Three measures were taken consecutively, removing the micrometer each time, and the

195 average was used in the analyses (intra-class correlation $\mathrm{R}>0.98$ ).

196

197 Measurement of blood metabolites

198 Carotenoid concentration in sera was measured by means of N-1000 NanoDrop

199 spectrophotometer at $450 \mathrm{~nm}$, at the maximum reflectance point for lutein, which is the main

200 circulating carotenoid found in passerines (Pérez-Rodríguez 2009). Total antioxidant capacity

201 (TAC) in sera was measured as described in (Erel 2004), implemented in Cobas INTEGRA

202 Chemistry autoanalyser. Recent studies point out that TAC is mostly representative of the water

203 soluble components of the antioxidative balance, and in combination with other fat-soluble

204 antioxidants provides a more complete image of the antioxidant system (Cohen and McGraw

205 2009). Uric acid and total protein were measured according to standard methods implemented on

206 a Cobas Integra 400 plus autoanalyzer with Roche reagents. Uric acid is a common circulating

207 antioxidant generated as a by-product of metabolism that accounts for an important portion of the

208 antioxidant capacity in blood, whereas total protein in sera is a standard diagnostic measure of

209 nutritional condition and may also reflect serum antioxidative activity (Roche et al 2008).

210 Repeatability for carotenoids, Uric acid, Total protein and TAC in sera, as assessed by the 
211 intraclass correlation of repeated blind measures of the same samples was higher than 0.97 for all

212 parameters.

213

214 Statistical methods

215 NDV-Ab concentration (expressed as the base 2 logarithm of the inverted dilution factor) from

216 11-day-old chicks was analyzed as dependent variable in a generalized linear mixed model

217 (GLMM), with normally distributed error and identity link. Mother and chick's treatments were

218 included as main effects and nestbox as a random factor in all models. Mother's final NDV-Ab

219 concentration (when caring the second brood), and time between treatment and sampling were

220 considered as covariates in both chick (7.72 \pm 0.19 days, range 5-12) and female (37.64 \pm 0.72

221 days, range=24-65) measurements, as differences arose due to hatching asynchrony and capture

222 success respectively. Likewise, in addition to main effects and the random factor, time between

223 measurements was included as covariate in the models for PHA response and mass change from

224 challenge to sampling, and otherwise models were identical. Chick survival from $4^{\text {th }}$ day of age

225 until fledging was analysed with a GLMM with binomially distributed error and logit link. Chick

226 and mother treatments were included as fixed factors, together with body mass at the time of

227 chick's treatment as covariate and nest as a random factor.

228 Afterwards, several parameters were tested in the previous models as covariates i.e. breeding

229 parameters (hatching date and clutch size), sex, biometric data (body mass and tarsus length) and

230 blood metabolites (TAC, Uric acid, Total protein and carotenoids). Different models were built

231 on NDV-Ab concentration in chicks and mothers; chick PHA response, mass change and chick

232 survival as dependent variables. Only breeding parameters were included as covariates in the

233 models on chick survival as no sex, biometric nor blood metabolite information were available 
234 from non-surviving chicks. Due to limited sample size, covariates and their interactions with the

235 main effects were included sequentially, and retained whenever significant or as judged by AIC

236 or Generalized $\mathrm{Chi}^{2}$ to avoid model over-parametrization. Initial and final models are presented

237 with the corresponding fit statistics and variable parameters (Estimate, F, DF and P values), and

238 non significant predictors are shown with their corresponding values when removed from the

239 final model (Supplementary material).

240 Least squared means are provided for categorical predictors and slope estimates for covariates \pm

241 standard error. Sample size varied among tests due to differential survival and available sera for

242 laboratory analyses (Table 1). Degrees of freedom for fixed effects were adjusted by the

243 between-within approximation that accounts for within-subject changes of any fixed effect and

244 divides the residual degrees accordingly (Schluchter and Elashoff 1990). Blood metabolite

245 variables were normalized by means of $\log (\mathrm{x}+1)$ transformation, and residuals from all models

246 on NDV-Ab concentration, PHA response and mass change followed normality. All analyses

247 were performed with procedure GLIMMIX SAS 9.2. (SAS Institute Inc. 2009). Raw data is

248 available at http://hdl.handle.net/10261/127825. 
250 Results

251

252 Female NDV-Ab concentration before challenge were similar between treatments $\left(\mathrm{F}_{1,14}=0.86\right.$;

$253 \mathrm{P}=0.37$ ), and no differences between experimental and control groups were found before

254 challenge for any of the physiological (Table 1) or reproductive parameters studied. As expected,

255 female NDV-Ab concentration increased significantly in vaccinated individuals with respect to

256 control ones (Control: $0.00 \pm 0.38$ vs. Vaccinated: $4.70 \pm 0.41 ; \mathrm{F}_{1,20}=70.67 ; \mathrm{P}<0.0001$ ) (Figure 1).

257 Neither pre-challenge NDV-Ab concentration $\left(\mathrm{F}_{1,12}=0.39 ; \mathrm{P}=0.54\right)$, days between vaccination

258 and re-sampling $\left(\mathrm{F}_{1,13}=0.07 ; \mathrm{P}=0.80\right)$, the physiological nor the reproductive parameters

259 considered (all $\mathrm{P}>0.5$ ) were related to post-challenge NDV-Ab concentration.

260

261 Chick NDV-Ab concentration at 11 days of age was independent of maternal treatment

$262\left(\mathrm{~F}_{1,17}=0.06 ; \mathrm{P}=0.81\right)$. Surprisingly, chick NDV-Ab concentration was independent from chick

263 treatment $\left(\mathrm{F}_{1,19}=3.83 ; \mathrm{P}=0.07\right)$, and the interaction with maternal treatment $\left(\mathrm{F}_{1,19}=0.52 ; \mathrm{P}=0.48\right)$.

264 Time between chick vaccination and sampling was not significant $\left(\mathrm{F}_{1,17}=0.26 ; \mathrm{P}=0.615\right)$.

265 However, when considering female NDV-Ab concentration as a covariate, the interaction

266 between female NDV concentration and chick's treatment appeared significant $\left(\mathrm{F}_{1,53}=6.61\right.$;

$267 \mathrm{P}=0.01$ ), together with days between chick and mother sampling (Estimate $0.324 \pm 0.121$;

$\left.268 \mathrm{~F}_{1,17}=7.20 ; \mathrm{P}=0.02\right)$. NDV-Ab concentration of vaccinated chicks tended to increase with female

269 NDV-Ab concentration, whereas this relation was not significant in control chicks (Control: -

$2700.11 \pm 0.14 ; \mathrm{t}_{18}=-0.81 ; \mathrm{P}=0.43$ vs. Vaccinated: $\left.0.23 \pm 0.13 ; \mathrm{t}_{18}=1.75 ; \mathrm{P}=0.09\right)$. Neither sex,

271 biometric measurements nor the breeding parameters were associated with NDV-Ab

272 concentration in chicks (all $\mathrm{P}>0.2$ ). Vaccinated chicks from vaccinated mothers developed higher 
273 specific humoral response, but only when originating from mothers exhibiting high NDV-Ab 274 titres (Figure 1).

275 On the other hand, when including blood metabolites as covariates on the previous model, only

276 carotenoids and TAC were related to chick NDV-Ab. Carotenoids decreased with increasing

277 NDV-Ab concentration in chicks, independently of the treatment (slope: $-1.35 \pm 0.47 ; F_{1,39}=8.11$;

$278 \mathrm{P}<0.01)$. However, the relation between NDV-Ab and TAC changed slightly according to the

279 chick treatment, the interaction being significant $\left(\mathrm{F}_{1,50}=5.95 ; \mathrm{P}=0.02\right)$. NDV-Ab concentration of

280 vaccinated chicks decreased with TAC, whereas the relation was not significant in control chicks

281 (Control: $-0.34 \pm 0.59 ; \mathrm{t}_{50}=-0.59 ; \mathrm{P}=0.56$ vs. Vaccinated: $\left.-2.08 \pm 0.45 ; \mathrm{t}_{50}=-4.66 ; \mathrm{P}<0.01\right)$. Chick's

282 concentration of NDV-Ab was negatively related to carotenoids in blood, and in the case of

283 vaccinated chicks also to TAC in blood (Figure 2. in Supplementary material). Uric acid or total

284 protein were unrelated to chick's NDV-Ab concentration (all $\mathrm{P}>0.1$ ).

PHA in chicks was unrelated to maternal $\left(\mathrm{F}_{1,9}=0.01 ; \mathrm{P}=0.93\right)$ or $\operatorname{chick}\left(\mathrm{F}_{1,21}=0.02 ; \mathrm{P}=0.88\right)$

287

288

289

290

291

292

293

294

295 treatments, even when accounting for chick body mass or change in body mass between

challenge and measurement (hereafter body mass change), and time between such measurements (all $\mathrm{P}>0.2$ ). Furthermore, none of the breeding parameters, biometric measurements nor blood metabolites considered were significantly related to PHA response (all $\mathrm{P}>0.09$ ). As expected, PHA variation in chicks was only significantly explained by body mass (slope: $-0.06 \pm 0.02$; $\left.\mathrm{F}_{1,29}=5.05 ; \mathrm{P}=0.03\right)$, as PHA response was lower the heavier the chick.

Body mass change in chicks from vaccination to sampling date (4 to 11 days of age) was independent of mother's and chick's NDV-Ab titres (all $\mathrm{P}>0.30$ ). Likewise, neither treatment, sex nor the blood metabolite variables were related to body mass change (all $\mathrm{P}>0.2$ ). As 
296 expected, elapsed days between vaccination and sampling (slope: $1.20 \pm 0.28 ; \mathrm{F}_{1,20}=18.23$;

$297 \mathrm{P}<0.01$ ) and tarsus length had a significant influence on body mass change (slope: $0.60 \pm 0.27$;

$298 \mathrm{~F}_{1,63}=4.96 ; \mathrm{P}=0.03$ ) (Table 1). House sparrow chicks with larger tarsi grew heavier independently

299 of their mother's or their own level of NDV-Ab, once the period between measurements was

300 accounted for.

$301 \quad$ Chick's survival from $4^{\text {th }}$ day of age until fledging was unrelated to neither the maternal

$302\left(\mathrm{~F}_{1,22}=0.76 ; \mathrm{P}=0.39\right)$, nor the chick's treatments $\left(\mathrm{F}_{1,23}=0.31 ; \mathrm{P}=0.59\right)$, and only body mass at the

303 time of vaccination was related to fledging success (slope: $0.49 \pm 0.11 ; \mathrm{F}_{1,89}=18.29 ; \mathrm{P}<0.01$ ). 
Discussion

305 House sparrow mothers developed a significant specific humoral response when challenged with

306 NDV vaccine before egg-laying, and as a result their offspring were more likely to develop a

307 specific humoral response when challenged with the same antigen. However, inter-individual

308 variation in maternal NDV-Ab was the main determinant of chick specific immune response to

309 NDV, as only chicks from mothers with high NDV Ab concentration significantly increased their

310 response to NDV vaccine (Figure 1).

311 Maternal Ab transfer may reflect a dynamic balance between the benefits of providing an early

312 specific protection and the costs of blocking the nestling's immune development (Garnier et al

313 2012). In our study, maternal Ab could not be detected in 11-day-old chicks by means of

314 standard diagnostic techniques, as no differences were found between control chicks from

315 vaccinated and control mothers. The results suggest that maternal Ab transmission in house

316 sparrows has a priming beneficial effect on the specific response of pre-fledging chicks (11 days

317 old), by stimulating the endogenous production of Ab when early exposed to same pathogens as

318 their mothers. This priming effect may be explained by a higher and/or faster antibody

319 production in chicks exposed to maternal Abs. However, disentangling such non-mutually

320 exclusive processes would require a detailed temporal monitoring of NDV-Ab response after

321 fledging, which is beyond the focus of this study.

322 On the other hand, mothers influence offspring immunity in other ways than transmission of

323 specific $\mathrm{Ab}$, by providing other components through the yolk or by modulating parental

324 investment. Therefore it is possible that the enhanced specific immune response in offspring

325 could result from other maternal effects than the transmission of specific Ab, or a combination of

326 them. However, the fact that chicks did not experience any change in growth rate or PHA- 
327 response which is a complex inflammatory and immune response suggests that maternal effects

328 were antigen-specific and not condition dependent (Ahmed et al 2007).

329 Maternal treatment per-se was ineffective in explaining offspring specific response, and only

330 when considering inter-individual variation in specific Ab levels such relationship was apparent.

331 This result can be explained by several non exclusive reasons. First, the different response

332 among breeding females to the experimental challenge, which led to a significant inter-individual

333 variation in circulating $\mathrm{Ab}$ level, could arise from the usual individual variation to experimental

334 vaccinations (Zinkernagel 2003). Second, some breeding females could have been previously

335 exposed to the virus in the wild (see Broggi et al 2013). Previous exposure would lead to

336 different maternal level of circulating $\mathrm{Ab}$ and in turn affect passive transmission of $\mathrm{Ab}$ to the

337 offspring. In fact, one control chick originating from a control mother presented NDV-Ab,

338 implying a natural exposure to the antigen (Figure 1). Second, it is possible that laying females

339 effectively transfer Ab only when their own systemic levels are above certain threshold

340 (Grindstaff 2010). Alternatively, it could be that maternally transmitted Ab do not persist long in

341 the nestling blood, and after 11 days of age they are not detectable anymore (Grindstaff et al

342 2003, Nemeth et al. 2008). The fact that breeding females were evaluated a few weeks after

343 being challenged, while their chicks were challenged and sampled after a few days may rend our

344 results conservative as it may be that maternal effects are most effectively transmitted earlier

345 after their challenge, or the effects appear after 11 days of chick age, but see (Garnier et al 2012).

346 The present results add to the accumulating evidence that exposure at different times during the

347 course of a decay of maternal antibodies, which can be short-lived in altricial species like

348 sparrows may affect chick immune response differently (Zinkernagel 2003). 
349 The few studies on maternally transmitted Ab on offspring specific immunity on wild avian

350 species are not conclusive as the effects often vary among host species, pathogens and timing of

351 exposure (Zinkernagel 2003; Hasselquist and Nilsson, 2012). The limited knowledge on the

352 persistence of maternal $\mathrm{Ab}$, the temporal pattern of $\mathrm{Ab}$ production and the long-term

353 consequences of maternal transmission limits our capacity to interpret these differences

354 (Boulinier and Staszewski 2008). Some studies report short-term enhancing effects (Grindstaff et

355 al 2006; Pihlaja et al 2006), while others have revealed a blocking effect (Gasparini et al 2009;

356 Staszewski et al 2007; Staszewski and Siitari 2010; Garnier et al 2012; Merrill and Grindstaff

357 2014). Interestingly, same host species can respond differently to maternally transmitted Ab

358 when exposed to varying antigens (Gasparini et al 2006; Addison et al 2010). Other studies have

359 found maternal transmission of $\mathrm{Ab}$ to buffer costs of an immune response (Grindstaff 2008),

360 whereas others found the effect of maternal Ab to be negligible (Nemeth et al 2008; King et al

361 2010). Finally, long-term effects of maternal Ab-transmission on the specific immune response

362 of chicks to antigens administrated on the previous breeding season to pre-laying mothers have

363 been reported blocking (Staszewski et al 2007), or enhancing the offspring specific response

364 (Reid et al 2006). In our study we found house sparrow mothers to enhance their offspring

365 specific humoral response during the pre-fledging period, but not their PHA response, implying

366 that specific Ab are transmitted without conditioning other components of their immune system

367 like those involved in the response to the PHA test. These contrasting results highlight the

368 varying effects that maternal Ab may have among hosts exposed to different pathogens, and the

369 need to analyse differences in the dynamics of antibody circulation, timing of exposure to

370 pathogens and the interaction with other maternally transferred components. 
371 Interspecific comparisons suggest that larger and longer lived species, which also experience

372 slower developmental times, may rely more strongly on the maternal effects that may be

373 transmitted in larger amounts and persist for longer periods (Garnier et al 2012, Ramos et al

374 2014). In line with this suggestion and in contrast with our results, it has been argued that

375 maternal transfer of $\mathrm{Ab}$ in fast-developing altricial species (e.g. house sparrow) may be limited

376 or not even play any relevant role as endogenous $\mathrm{Ab}$ production is achieved soon after hatching,

377 leaving maternal $\mathrm{Ab}$ a too short time-frame to be effective (King et al. 2010). Alternatively,

378 maternal Ab may be effectively transmitted and still remain undetected, as they may persist for a

379 too short time period or because of practical constraints as good samples are difficult to obtain

380 from hatchlings of those small-sized species (Lozano \& Ydenberg 2002, Nemeth et al 2008,).

381 Furthermore, methodological differences among studies may partly explain the varying results

382 on the effects of the maternal transmission of $\mathrm{Ab}$ on offspring specific immunity. Although most

383 studies tested the effects by studying both mothers and offspring exposed to the same antigen,

384 this was not always the case (e.g. Gasparini et al 2006; Pihlaja et al 2006; King et al 2010). Our

385 results suggest that maternal transfer of antibodies in the house sparrow is important enough to

386 prime chick specific immunity, despite not being detectable by usual immunological techniques.

387 The effect of maternally transmitted Ab appears to be host-pathogen specific, and dependent on

388 the development of host humoral immunity at the time of infection. Furthermore, such effect

389 could be of a hormetic nature (Costantini et al 2010), irrelevant under certain threshold and

390 priming or blocking according to the different concentration of $\mathrm{Ab}$ transmitted and the individual 391 condition (Lemke et al 2004). 
393 The ontogeny of the constitutive immunity is complex, influenced by both maternal and 394 endogenous factors, with long term implications for the individual development and overall 395 immunity (Butler and McGraw 2011; van der Most et al 2011; Arriero et al 2013). Maternal 396 transfer of specific Ab could benefit offspring by enhancing the specific humoral response while 397 permitting growth rate to be maintained (Grindstaff 2008), as found in this study. However, we 398 found chicks developing stronger specific humoral response experienced decreased carotenoid 399 levels and antioxidative capacity in blood. In addition to Ab, parent females transfer carotenoids 400 and antioxidants to their eggs according to diverse endogenous and environmental factors such 401 as mate attractiveness (Saino et al 2002), own condition (Hammouda et al 2012), pathogen abundance (Gasparini et al 2001) or predation risk (Morosinotto et al 2013). We found no 403 differences in blood carotenoids or TAC measured in females either at challenge or post404 challenge sampling times in relation to any of the treatments or NDV-Ab concentration, 405 suggesting that variation in chick's levels arose from chick's own physiological adjustments 406 rather than differential maternal transmission. Several studies have shown costly aspects of 407 immunity in terms of antioxidative balance and carotenoid levels. Interestingly, other studies 408 found carotenoid levels to be positively related to humoral response but unrelated to PHA response (Bédécarrats and Leeson 2006). Carotenoids appear to compensate costly immune 410 responses to pathogens (Ewen et al 2009), or become depleted the stronger an immune response 411 (Saino et al 2003). In our study, house sparrow offspring fledged independently of their 412 treatment or NDV-Ab concentration, suggesting that supposed advantages of enhanced specific 413 humoral response, or costs of decreased antioxidative capacity and carotenoids in blood are to be 414 experienced on a longer-term. However, the final cost-benefits balance derived from maternal 
415 transmission of antibodies will depend on the rate of exposure to pathogens, and the fitness costs

416 derived from pathogen exposure in chicks with and without maternal antibodies.

417

418 In summary, we found that the specific humoral response to NDV in 11-day-old house sparrow 419 chicks is enhanced by maternal exposure to NDV, most likely through passive transmission of 420 specific Ab. However, nestlings investing in specific NDV-Ab production present lower 421 carotenoid concentration and impaired antioxidative capacity in blood, suggesting that maternal 422 priming of specific humoral response can be beneficial but may come to a physiological cost in 423 pre-fledging small altricial species.

425 Acknowledgements

426 We thank Marta Aliseda, Juan Luis Barroso, Oscar González, Cristina Pérez, Airam Rodríguez 427 and Matthias Vögeli for their help during the fieldwork. We are indebted to Francisco Miranda 428 and Olaya García for their work with sample analysis at the Ecophysiology laboratory in EBD. 429 Plácido and Maribel from la Cañada de los Pájaros gently allowed us to work in their property. 430 Albert Pagès and HIPRA personnel provided advice and technical support on NDV vaccine use 431 and analysis. Jan-Åke Nilsson, Romain Garnier and one anonymous referee provided valuable 432 comments on earlier versions of the manuscript.

434 Data Accessibility

Data deposited at http://hdl.handle.net/10261/127825 
437 References

438 Adamo SS (2004) How should behavioural ecologists interpret measurements of immunity? Anim Behav 68:1443-1449

440

441

Addison B, Ricklefs RE, Klasing KC (2010) Do maternally derived antibodies and early immune experience shape the adult immune response? Funct Ecol 24:824-829

Ahmed KA, Saxena VK, Ara A, Singh KB, Sundaresan NR, Saxena M, Rasool TJ (2007) Immune response to Newcastle disease virus in chicken lines divergently selected for cutaneous hypersensitivity. Int J Immunogen 34:445-455

Alexander DJ (2009) Ecology and epidemiology of Newcastle disease. In: Capua I, Alexander DJ (eds) Avian Influenza and Newcastle Disease. A Field and Laboratory Manual. Springer-Verlag, Milan, pp 19-26

Anderson TR (2006) Biology of the ubiquitous house sparrow. Oxford University Press, New York.

Ardia DR (2005) Individual quality mediates trade-offs between reproductive effort and immune function in tree swallows. J Anim Ecol 74:517-524

Ardia DR, Parmentier HK, Vogel LA (2011) The role of constraints and limitation in driving individual variation in immune response. Funct Ecol 25:61-73

Arriero E, Majewska A, Martin TE (2013) Ontogeny of constitutive immunity: maternal vs. endogenous influences. Funct Ecol 27:472-478 
456 Bédécarrats GY, Leeson S (2006) Dietary lutein influences immune response in laying hens. J

457

458

459

460

461

462

463

464

465

466

467

468

469

470

471

472

473

474

Appl Poult Res 15:183-189

Blount JD, Metcalfe NB, Birkhead TR, Surai PF (2003) Carotenoid modulation of immune function and sexual attractiveness in zebra finches. Science 300:125-127

Boulinier T, Staszewski V (2008) Maternal transfer of antibodies: raising immuno-ecology issues. Trend Ecol Evol 23:282-288

Broggi J, García O, Miranda F, Pagès A, Soriguer RC, Figuerola J (2013) Immune response to Newcastle disease virus vaccination in a wild passerine. J Wild Dis 49:1004-1008

Butler MW, McGraw KJ (2011) Developmental immune history affects adult immune function but not carotenoid-based ornamentation in mallard ducks. Funct Ecol 26:406-415

Cohen AA, McGraw KJ (2009) No simple measures for antioxidant status in birds: complexity in inter- and intraspecific correlations among circulating antioxidant types. Funct Ecol 23:310-320

Costantini D, Metcalfe NB, Monaghan P (2010) Ecological processes in a hormetic world. Ecol Lett 13:1435-1447

Costantini D, Møller AP (2009) Does immune response cause oxidative stress in birds? A metaanalysis. Comp Biochem Physiol A 153:339-344

Dowling DK, Simmons LW (2009) Reactive oxygen species as universal constraints in lifehistory evolution. Proc R Soc Lond B 276:1737-1745 
475 476

477

Elazab MFA, Fukushima Y, Horiuchi H, Matsuda H, Furusawa S (2009) Prolonged suppression of chick humoral immune response by antigen specific maternal antibody. J Vet Med Sci $71: 417-424$

Erel O (2004) A novel automated direct measurement method for total antioxidant capacity using a new generation, more stable ABTS radical cation. Clin Biochem 37:277-285

Ewen JG, Thorogood R, Brekke P, Cassey P, Karadas F, Armstrong DP (2009) Maternally invested carotenoids compensate costly ectoparasitism in the hihi. Proc Nat Acad Sci USA 106:12798-12802

Forsman AM, Vogel LA, Sakaluk SK, Grindstaff JL, Thompson CF (2008) Immune-challenged house wren broods differ in the relative strengths of their responses among different axes of the immune system. J Evol Biol 21:873-878

Fridolfsson AK, Ellegren H (1999) A simple and universal method for molecular sexing of nonratite birds. J Avian Biol 30:116-121

Garnier R, Ramos R, Staszewski V, Militao T, Lobato E, González-Solís J, Boulinier T (2012) Maternal antibody persistence: a neglected life-history trait with implications from albatross conservation to comparative immunology. Proc R Soc Lond B 279:2033-2041

Gasparini J, McCoy KD, Haussy C, Tveraa T, Boulinier T (2001) Induced maternal response to the Lyme disease spirochaete Borrelia burgdorferi sensu lato in a colonial seabird, the kittiwake Rissa tridactyla. Proc R Soc Lond B 268:647-650 
494 Gasparini J, McCoy KD, Staszewski V, Haussy C, Boulinier T (2006) Dynamics of anti-Borrelia 495 antibodies in Black-legged Kittiwake (Rissa tridactyla) chicks suggest maternal 496 educational effect. Can J Zool 84:623-627

497

498

499 500

501

502

503

504

505

506

507

508

509

510

511

512

Gasparini J, Piault R, Bize P, Roulin A (2009) Pre-hatching maternal effects inhibit nestling humoral immune response in the tawny owl Strix aluco. J Avian Biol 40:271-278

Grindstaff JL (2008) Maternal antibodies reduce costs of an immune response during development. J Exp Biol 211:654-660

Grindstaff JL (2010) Initial levels of maternally derived antibodies predict persistence time in offspring circulation. J Ornithol 151:423-428

Grindstaff JL, Brodie III ED, Ketterson ED (2003) Immune function across generations: integrating mechanism and evolutionary process in maternal antibody transmission. Proc R Soc Lond B 270:2309-2319

Grindstaff JL, Hasselquist D, Nilsson JÅ, Sandell M, Smith HG, Stjernman M. (2006) Transgenerational priming of immunity: maternal exposure to a bacterial antigen enhances offspring humoral immunity. Proc R Soc Lond B 273:2551-2557

Groothuis TGG, Schwabl HG (2008) Hormone-mediated maternal effects in birds: mechanisms matter but what do we know of them? Phil Trans R Soc Lond B 363:1647-1661

Hammouda A, Selmi S, Pearce-Duvet J, Chokri MA, Arnal A, Gauthier-Clerc M, Boulinier T (2012) Maternal antibody transmission in relation to mother fluctuating asymmetry in a 
515 Hasselquist D, Nilsson J (2009) Maternal transfer of antibodies in vertebrates: transgenerational effects on offspring immunity. Phil Trans R Soc Lond B 364:51-60

517 Hasselquist D, Nilsson JÅ (2012) Physiological mechanism mediating costs of immune responses: what can we learn from studies of birds? Anim Behav 83:1303-1312

519

520

521

522

523

524

525

526

527

528

529

530

531

Hasselquist D, Tobler M, Nilsson JÅ (2012) Maternal modulation of offspring immune function in vertebrates. In: Demas GE, Nelson RJ (eds) Ecoimmunology. Oxford University Press, Oxford.

Hochachka WM, Dhondt AA (2000) Density-dependent decline of host abundance resulting from a new infectious disease. Proc Nat Acad Sci USA 97:5303-5306

Hõrak P, Zilmer M, Saks L, Ots I, Karu U, Zilmer K (2006) Antioxidant protection, carotenoids and the costs of immune challenge in greenfinches. J Exp Biol 209:4329-4338

Iseri VJ, Klasing KC (2013) Dynamics of the systemic components of the chicken (Gallus gallus domesticus) immune system following activation by Escherichia coli; implications for the costs of immunity. Dev Comp Immun 40:248-257

King MO, Owen JP Schwabl HG (2010) Are maternal antibodies really that important? Patterns in the immunologic development of altricial passerine house sparrows (Passer domesticus). PLoS ONE 5:e9639 
532 Koutsos EA, García López JC, Klasing KC (2007) Maternal and dietary carotenoids interactively

533 affect cutaneous basophil responses in growing chickens (Gallus gallus domesticus).

$534 \quad$ Comp Biochem Physiol B 147:87-92

535 Lazzaro BP, Little TJ (2009) Immunity in a variable world. Phil Trans R Soc B 364:15-26

536 Lemke H, Coutinho A, Lange H (2004) Lamarckian inheritance by somatically acquired

537 maternal IgG phenotypes. Trends Immun. 25:180-186

538 Lozano GA, Ydenberg RC (2002) Transgenerational effects of maternal immune challenge in 539 tree swallows (Tachycineta bicolor). Can J Zool 80:918-925

540 McGraw KJ, Ardia DR (2003) Carotenoids, immunocompetence, and the information content of $541 \quad$ sexual colors: an experimental test. Am Nat 162:704-712

542 Midamegbe A, Grégoire A, Staszewski V, Perret P, Lambrechts MM, Boulinier T, Doutrelant C 543 (2013) Female blue tits with brighter yellow chests transfer more carotenoids to their $544 \quad$ eggs after an immune challenge. Oecologia 173:387-397.

545 Merrill L, Grindstaff JL (2014) Maternal antibody transfer can lead to suppression of humoral 546 immunity in developing zebra finches (Taeniopygia guttata). Physiol Biochem Zool

547 87:740-751

Monaghan P, Metcalfe NB, Torres R (2009) Oxidative stress as a mediator of life history trade549 offs: mechanisms, measurements and interpretation. Ecol Lett 12:75-92 present. Phil Trans R Soc Lond B 364:1035-1038 
552 Morosinotto C, Ruuskanen S, Thomson RL, Siitari H, Korpimäki E, Laaksonen T (2013)

553 Predation risk affects the levels of maternal immune factors in avian eggs. J Avian Biol

554 $44: 427-436$.

555 Navarro C, Marzal A, De Lope F, Møller AP (2003) Dynamics of an immune response in house 556 sparrows Passer domesticus in relation to time of day, body condition and blood parasite 557 infection. Oikos 101:291-298

558 Nemeth NM, Oesterle PT, Bowen RA (2008) Passive immunity to west nile virus provides limited protection in a common passerine species. Am J Trop Med Hyg 79:283-290

560

561

562

563

564

565

566

567

568

569

570

571

572
OIE (2015) Manual of Diagnostic Tests and Vaccines for Terrestrial Animals 2015. Available from http://www.oie.int/en/international-standard-setting/terrestrial-manual/accessonline/ (accessed July 2015)

Pérez-Rodríguez L (2009) Carotenoids in evolutionary ecology: Re-evaluating the antioxidant role. BioEssays 31:1116-1126

Pihlaja M, Siitari H, Alatalo RV (2006) Maternal antibodies in a wild altricial bird: effects on offspring immunity, growth and survival. J Anim Ecol 75:1154-1164

Ramos R, Garnier R, González-Solís J, Boulinier T (2014) Long antibody persistence and transgenerational transfer of immunity in a long-lived vertebrate. Am Nat 184:764-776

Reid JM, Arcese P, Keller LF, Hasselquist D (2006) Long-term maternal effect on offspring immune response in song sparrows Melospiza melodia. Biol Lett 2:573-576

Roche M, Rondeau P, Singh NR, Tarnus E, Bourdon E (2008) The antioxidant properties of serum albumin. FEBS Lett 582:1783-1787. 
573 Royle NJ, Surai PF, Hartley IR (2003) The effect of variation in dietary intake on maternal

574 deposition of antioxidants in zebra finch eggs. Funct Ecol 17:472-481

575 Saino N, Ferrari RP, Martinelli R, Romano M, Rubolini D, Møller AP (2002) Early maternal

576 effects mediated by immunity depend on sexual ornamentation of the male partner. Proc

$577 \quad$ R Soc Lond B 269:1005-1009

578 Saino N, Ferrari, RP, Romano M, Martinelli R, Møller AP (2003) Experimental manipulation of 579 egg carotenoids affects immunity of barn swallow nestlings. Proc R Soc Lond B $580 \quad 270: 2485-2489$

581 Schluchter MD, Elashoff JD (1990) Small-sample adjustments to tests with unbalanced repeated measures assuming several covariance structures. J Stat Comp Simul 37:69-87

583

584

Simons MJP, Cohen AA, Verhulst S (2012) What does carotenoid-dependent coloration tell? plasma carotenoid level signals immunocompetence and oxidative stress state in birds-a meta-analysis. PLoS ONE 7:e43088

Smits JE, Bortolotti GR, Tella JL (1999) Simplifying the phytohaemagglutinin skin-testing technique in studies of avian immunocompetence. Funct Ecol 13:567-572

Speakman JR, Garratt M (2014) Oxidative stress as a cost of reproduction: Beyond the simplistic trade-off model. BioEssays 36:93-106

Staszewski V, Gasparini J, McCoy KD, Tveraa T, Boulinier T (2007) Evidence of an interannual effect of maternal immunization on the immune response of juveniles in a long-lived colonial bird. J Anim Ecol 76:1215-1223

Staszewski V, Siitari H (2010) Antibody injection in the egg yolk: maternal antibodies affect humoral immune response of the offspring. Funct Ecol 24:1333-1341 
595 van der Most PJ, de Jong B, Parmentier HK, Verhulst S (2011) Trade-off between growth and 596 immune function: a meta-analysis of selection experiments. Funct Ecol 25:74-80

597 Viney ME, Riley,EM, Buchanan KL (2005) Optimal immune responses: immunocompetence $598 \quad$ revisited. Trends Ecol Evol 20:665-669

599 Vinkler M, Bainova H, Albrecht T (2010) Functional analysis of the skin-swelling response to 600 phytohaemagglutinin. Funct Ecol 24:1081-1086

601 Zinkernagel RM (2003) On natural and artificial vaccinations. Annu Rev Immunol, 21:515-546 


\section{Table $\mathbf{1}$ (on next page)}

Body mass and blood metabolite profiles for the different experimental treatments with their corresponding sample size

Body mass increase of house sparrow chicks from vaccination at four days of age to blood sampling at ten days of age, and body mass of house sparrow mothers according to the different treatments ( $C=$ control, $V=V a c c i n a t e d)$. Change is mass is expressed as the least squared means from a GLM with treatments and the number of days elapsed as a covariate. Different treatments correspond to the combination of mother (Mo) and chicks' (Ch) treatment: control chicks from control mothers ( $\mathrm{MoC} \mathrm{ChC}$ ); control chicks from vaccinated mothers (MoV ChC); vaccinated chicks from control mothers (MoC ChV); and vaccinated chicks from vaccinated mothers (MoV ChV). Mean values with the corresponding standard error (SE) are provided for the different blood metabolite parameters (Total antioxidant capacity (TAC); Carotenoids (CAR); Total protein (TPR); Uric Acid (UAC)), for chicks and mothers on different treatments. Sample sizes are given within parentheses. 


\begin{tabular}{cccccc}
\hline Chicks & Mass change \pm SE $(\mathrm{g})$ & TAC \pm SE $(\mu \mathrm{mol} / \mathrm{L})$ & $\mathrm{CAR} \pm \mathrm{SE}(\mathrm{mg} / \mathrm{L})$ & $\mathrm{TPR} \pm \mathrm{SE}(\mathrm{mg} / \mathrm{dL})$ & $\mathrm{UAC} \pm \mathrm{SE}(\mathrm{mg} / \mathrm{dL})$ \\
\hline MoC ChC & $6.98 \pm 0.70(21)$ & $931.34 \pm 111.05(21)$ & $15.62 \pm 2.26(16)$ & $2.23 \pm 0.16(21)$ & $7.03 \pm 0.62(21)$ \\
$\mathrm{MoC} \mathrm{ChV}$ & $7.14 \pm 0.63(25)$ & $721.07 \pm 103.88(24)$ & $14.70 \pm 2.02(20)$ & $2.14 \pm 0.15(24)$ & $6.82 \pm 0.58(24)$ \\
MoV ChC & $5.52 \pm 0.73(19)$ & $890.97 \pm 116.75(19)$ & $10.38 \pm 2.26(16)$ & $2.10 \pm 0.17(19)$ & $6.81 \pm 0.65(19)$ \\
MoV ChV & $4.99 \pm 0.67(23)$ & $888.00 \pm 111.05(21)$ & $12.19 \pm 2.08(19)$ & $2.36 \pm 0.17(21)$ & $6.58 \pm 0.62(21)$ \\
\hline Mothers & Mass $\pm \mathrm{SE}(\mathrm{g})$ & & & & \\
\hline Mo C & $26.25 \pm 0.46(13)$ & $1101.48 \pm 139.12(12)$ & $8.46 \pm 3.19(12)$ & $3.09 \pm 0.22(12)$ & $14.15 \pm 1.78(12)$ \\
Mo V & $26.11 \pm 0.48(12)$ & $963.58 \pm 145.31(11)$ & $10.16 \pm 2.88(12)$ & $3.02 \pm 0.23(11)$ & $14.84 \pm 1.85(11)$ \\
\hline
\end{tabular}




\section{1}

Concentration of NDV antibodies in house sparrow with respect to their mother, for each experimental treatment

Newcastle disease virus (NDV) antibody titres for house sparrow chicks and their mothers, in relation to the different experimental treatments $(N=1-20)$. Sizes of the circles correspond to sample size. Antibody titres are expressed as the log of the inverse of the dilution factor.

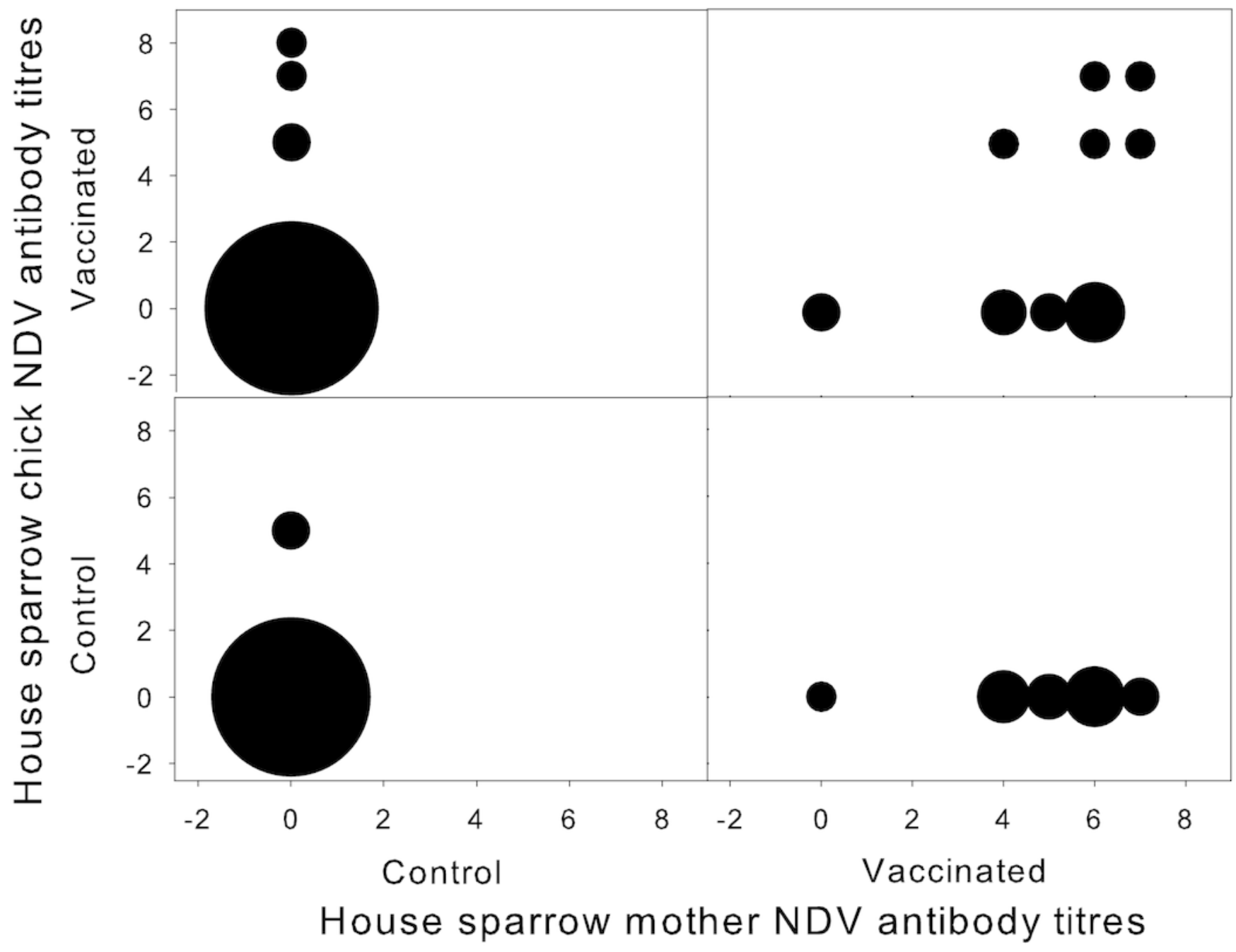

